\title{
Hämophiliebehandlung in Österreich
}

\author{
Ingrid Pabinger - Max Heistinger - Wolfgang Muntean - Sylvia-Elisabeth Reitter-Pfoertner • \\ Sabine Rosenlechner - Thomas Schindl - Gerhard Schuster . \\ Werner Streif · Katharina Thom · Christoph Male
}

Eingegangen: 16. Januar 2015 / Angenommen: 16. September 2015 / Online publiziert: 2. November 2015 (C) Die Autor(en) 2015. Dieser Artikel ist auf Springerlink.com mit Open Access verfügbar

Zusammenfassung Die vorliegende Leitlinie, die unter Patronanz der Österreichischen Hämophiliegesellschaft (ÖHG) sowie der Österreichischen Gesellschaft für Kinder- und Jugendheilkunde (ÖGKJ) und der Österreichischen Gesellschaft für Hämatologie und Onkologie (OeGHO) erstellt wurde, soll einen praxisnahen Leitfaden für die Diagnostik und Therapie von Hämophiliepatienten in Österreich darstellen. In der Hämophilietherapie gibt es wenige vergleichende Interventionsstudien, und die Empfehlungen haben einen meist niedrigen Evidenzgrad. Als wesentliche Grundlage dieser Leitlinie dienten neue, internationale Guidelines der „World Federation of Hemophilia" aus dem Jahr 2013. Diese wurde den nationalen Gegebenheiten und Erfahrungen angepasst. Behandelt werden Themen wie Diagnostik, Verlaufskontrollen, medikamentöse Therapieoptionen, Prophylaxe und Therapie bei Kindern und Erwachsenen, weiters mögliche Probleme bei Konduktorinnen und spezielle Aspekte wie Heimtherapie, Optionen für venöse Zugänge,
Umgang mit verschiedenen Formen von Traumata, Blutungen und Eingriffen einschließlich Zahnextraktionen und das Thema Hemmkörper und dessen Behandlung.

Schlüsselwörter Hämophilie · Leitlinien · Gerinnungsfaktorkonzentrate $\cdot$ Desmopressin

\section{Treatment of haemophilia in Austria}

Summary This guideline which is endorsed by the Austrian Society of Haemophilia, the Austrian Society of Paediatrics, and the Austrian Society of Haematology \& Medical Oncology is intended to give a clear and practical guidance for diagnosing and treating haemophilia in Austria. In the treatment of haemophilia there are few controlled interventional trials, and recommendations usually have a rather low level of evidence.

The main basis for this paper are the new international guidelines by the World Federation of Hemophilia, pub-

Univ.-Prof. Dr. I. Pabinger ( $\square)$ · S.-E. Reitter-Pfoertner, MD Klinische Abteilung für Hämatologie und Hämostaseologie, Universitätsklinik für Innere Medizin I,

Medizinische Universität Wien,

Währinger Gürtel 18-20,

1090 Wien, Österreich

E-Mail: ingrid.pabinger@meduniwien.ac.at

M. Heistinger, MD

1. Medizinische Abteilung, LKH Klagenfurt,

Klagenfurt, Österreich

W. Muntean, MD

Universitätsklinik für Kinder- und Jugendheilkunde,

Medizinische Universität Graz,

Graz, Österreich

S. Rosenlechner, MD

Klinik Prof. Schedel,

Kellberg bei Passau, Deutschland

\section{T. Schindl, MSc}

Österreichische Hämophilie Gesellschaft,

Wien, Österreich

G. Schuster, MD

Blutzentrale des Roten Kreuzes,

Linz, Österreich

\section{W. Streif, MD}

Department für Kinder- und Jugendheilkunde,

Medizinische Universität Innsbruck,

Innsbruck, Österreich

K. Thom, MD · C. Male, MD

Universitätsklinik für Kinder- und Jugendheilkunde,

Medizinische Universität Wien,

Wien, Österreich 
lished in 2013. These were adapted according to the local situation and experience.

Covered topics are diagnostics, control visits, pharmacological treatment options, prophylaxis and treatment in children and adults, possible problems arising in haemophilia carriers and special aspects like home therapy, options for venous catheters, management of various traumas, bleedings and interventions, including dental procedures, and last not least inhibitors and their treatment.

Keywords Haemophilia · Guidelines · Clotting factor concentrates $\cdot$ Desmopressin

Die vorliegende Leitlinie ist die erste zur Behandlung der angeborenen Hämophilie in Österreich. Unter „Behandlung" ist in diesem Zusammenhang sowohl die prophylaktische als auch die therapeutische Gabe von Blutgerinnungsfaktor-Konzentraten (in weiterer Folge kurz „Faktorkonzentrate“ genannt, weiters auch alle anderen für die Hämophilie relevanten prophylaktischen und therapeutischen Maßnahmen) zu verstehen.

Für die „Evidence-Based-Medicine“(EBM")-konforme Wertung der hier getroffenen Empfehlungen wird das System der Evidenzlevels des „Oxford Centre for Evidence-Based Medicine“ 2011 herangezogen, das auch in den rezenten Leitlinie der „World Federation of Hemophilia" (WFH) verwendet wurde (s. Appendix). Die Evidenz wurde in der vorliegenden Leitlinie nicht neu bewertet, sondern von den Leitlinien der WFH übernommen [1]. Die Leitlinie wurde durch Mitglieder des wissenschaftlichen Beirates der österreichischen Hämophiliegesellschaft unter der Patronanz zweier medizinischer Fachgesellschaften, der Österreichischen Gesellschaft für Hämatologie \& Medizinische Onkologie (OeGHO) und der Österreichischen Gesellschaft für Kinder- und Jugendheilkunde (ÖGKJ) erstellt. Sie soll den Stand des Wissens der Hämophilie-Behandlung beschreiben und damit auch in der Hämophilie nicht so erfahrenen Kollegen als Richtschnur dienen.

Bei der Hämophilie handelt es sich um eine seltene Erkrankung („orphan disease“). Mit der Publikation dieser Leitlinie liegt nun erstmals ein Konsensus-Dokument für Österreich vor, das den Stand des Wissens in der Diagnostik und Behandlung der Hämophilie zusammenfasst und als gemeinsame Referenz für eine angemessene medizinische Betreuung von Hämophiliepatienten dienen soll. Über den angeführten Grad an wissenschaftlicher Evidenz hinaus kommt dem Dokument besondere Wertigkeit $\mathrm{zu}$, weil Patienten und ihre Interessensvertretung aktiv in seine Erstellung einbezogen wurden. Besonders bei schwerwiegenden seltenen Erkrankungen sind ein akkordiertes Vorgehen und ein vertrauensvolles Verhältnis zwischen Arzt und Patient wichtig. Durch die Möglichkeit der Heimbehandlung werden dem Patienten diagnostische („Ist dies eine Blutung?") und therapeutische (Selbstinjektion) Aufgaben übertragen. Die Leitlinie soll zu einer gleichmäßig hohen Qualität der
Tab. 1 Schweregrade der Hämophilie A/B nach Faktoraktivität. (Quelle: [1])

\begin{tabular}{l|l|}
\hline Faktoraktivitäta & \multicolumn{1}{|c|}{ Schweregrad } \\
\hline$<1 \%$ & Schwer \\
\hline $1-5 \%$ & Mittelschwer \\
\hline $5-40 \%$ & Leicht \\
\hline $\begin{array}{l}\text { aBezogen auf die bei Gesunden messbaren Normalwerte; in der Literatur } \\
\text { werden auch } \mathrm{U} / \mathrm{ml} \text { angegeben; } 1 \% \text { entspricht } 0,01 \mathrm{U} / \mathrm{ml}\end{array}$ \\
\hline
\end{tabular}

Hämophiliebehandlung in Hämophiliezentren, aber auch nicht-spezialisierten Spitälern und Arztpraxen in ganz Österreich beitragen.

\section{Definitionen}

Bei der Hämophilie handelt es sich um eine X-chromosomal vererbte Blutgerinnungsstörung, die auf Mutationen der für FVIII bzw. FIX kodierenden Gene beruht [1]. Bei Hämophilie A ist der Faktor VIII betroffen, die Inzidenz liegt bei 1:10.000 Geburten. Bei Hämophilie B ist der Faktor IX betroffen, die Inzidenz liegt bei 1:50.000. Differentialdiagnostisch sind die verschiedenen Formen des vWS abzugrenzen sowie die durch Autoantikörper verursachte „erworbene Hämophilie“ bei genetisch nicht-hämophilen Personen.

Tabelle 1 stellt die Schweregrade der Hämophilie anhand der beim individuellen Patienten im Plasma vorhandenen Faktoraktivität dar und unterteilt diese in schwer, mittelschwer und leicht. Die frühere Abgrenzung einer Subhämophilie mit einer Faktoraktivität von $>15 \%$ wird nicht mehr verwendet. Der obere Grenzwert wurde in Übereinstimmung mit den internationalen Leitlinien mit $40 \%$ definiert [1]. Jedoch variiert dieser Grenzwert in unterschiedlichen Publikationen zwischen 25 und 50\% (dem unteren Grenzwert des Normalbereichs) [2]. In der FVIII-Mutationsdatenbank sind einige Hämophiliemutationen, die mit einer FVIII-Aktivität zwischen 40 und $50 \%$ einhergehen, beschrieben [3].

Der Faktor VIII als Akutphasenprotein kann bei besonderen klinischen Situationen (z. B. bei Entzündungen oder nach einer Operation oder Blutung) bei Patienten mit leichter Hämophilie mitunter auf über 40-60\%, also bis in den Normbereich, ansteigen. Diese Aspekte sind bei Diagnosestellung oder beim Ausschluss einer Hämophilie in Erwägung zu ziehen.

\section{Diagnostik}

\section{Initialdiagnostik}

Die diagnostische Evaluierung von Patienten mit Verdacht auf Hämophilie, bei denen eine Blutungsneigung festgestellt wurde, beginnt mit der Familien- und Eigenanamnese des Patienten, wobei auf das Auftreten von Gerinnungsstörungen in der Familie besonderer Wert gelegt wird. Bei genetisch potentiell hämophilen Kin- 
Tab. 2 Laboruntersuchungen für die Initialdiagnostik. (Quelle: Autoren)

\begin{tabular}{l}
$\begin{array}{l}\text { Gerinnungsglobaltests: PTZ, aPTT (faktorensensitive aPTT), TZ, Fibrinogen } \\
\text { (funktionell nach Clauss) }\end{array}$ \\
Gerinnungsfaktor-Einzelanalyse (insbesondere FVIIIc/FIXC [koagulometrisch, \\
ev. zusätzlich chromogen], Von-Willebrand-Diagnostik (vor allem zum Aus- \\
schluss eines Typ Normandie) [56]) \\
\hline $\begin{array}{l}\text { Ausschluss von Inhibitoren gegen FVIII und FIX (Vorgehen z. B. nach } \\
\text { [57]: Tauschversuch usw.), Lupus-Antikoagulans, Antikoagulanzienwirkung }\end{array}$ \\
\hline Blutbild komplett, Leber- und Nierenfunktionsparameter, CRP \\
\hline Blutgruppenserologie \\
\hline Infektionsstatus (Serologie Hepatitis A, B, C; HIV) \\
\hline $\begin{array}{l}\text { Mutationsdiagnostik (Diagnosesicherung, Einschätzung eines Hemmkörper- } \\
\text { risikos, Konduktorinnenstatus) }\end{array}$
\end{tabular}

dern und Jugendlichen erfolgt die Diagnostik auch ohne Blutungsneigung zum Ausschluss oder zur Bestätigung einer Hämophilie. Tabelle 2 listet die wichtigsten Laboruntersuchungen für die initiale Diagnostik auf.

Nach Diagnosestellung sollte - laut Empfehlung der WFH - unverzüglich die Anbindung des betroffenen Patienten an ein Hämophiliezentrum erfolgen. Idealerweise sollte es sich dabei um ein „Comprehensive Care Haemophilia Centre" handeln, in dem eine multidisziplinäre Betreuung von Hämophiliepatienten möglich ist. Europäische Standards für „Comprehensive Care Haemophilia Centers“ (CCHC) und „Haemophilia Treatment Centers" (HTC) wurden durch eine interdisziplinäre Arbeitsgruppe 2008 definiert [4]. Das Hämophiliezentrum des AKH (Betreuung von Kindern und Erwachsenen) ist seit 2014 als CCHC zertifiziert.

\section{Verlaufskontrollen}

Verlaufskontrollen setzen sich aus folgenden Teiluntersuchungen zusammen:

- Körperliche Untersuchung einschließlich des klinischen Gelenksstatus: Die Erhebung standardisierter physikalischer Scores wird empfohlen, da sie eine Einschätzung des muskuloskelettalen Systems und Verlaufskontrollen von dessen Zustand erlauben. Scores zur Evaluierung können von der WFH-Website (www.wfh.org) heruntergeladen werden [5].

- Laboruntersuchungen: FVIII/IX-Talspiegel, eventuell Recovery (= Faktorspiegel 30-60 min nach Faktorsubstitution) und weitere FVIII/IX-Zeitverlaufsbestimmungen (Pharmakokinetik); Hemmkörper (nach Bethesda-Methode), Infektionsstatus; Blutbild, Leber- und Nierenfunktionsproben

- Nach Bedarf bildgebende Untersuchungen von Gelenken (Ultraschall, Nativröntgen, Magnetresonanztomographie)

- Die Kontrolluntersuchungen im CCHC oder HTC sollten zur Anpassung des Behandlungsplans (Dosis, Frequenz) genützt werden.
Bei schwerer und mittelschwerer Hämophilie sollten Kontrolluntersuchungen bei bisher unbehandelten Kindern („Previously Untreated Patients“ - PUP) bis zum 50. Expositionstag zunächst alle fünf bis zehn FVIII/ IX-Expositionstage erfolgen, danach alle drei bis sechs Monate.

Bei leichter Hämophilie sollen die Kontrolluntersuchungen je nach Blutungs- und Behandlungsfrequenz erfolgen, mindestens jedoch einmal jährlich.

\section{Medikamentöse Therapieoptionen}

An Medikamenten zur Behandlung der Hämophilie stehen zur Verfügung:

- Faktorkonzentrate

- Desmopressin

- Antifibrinolytika (Tranexamsäure)

- Analgetika

- Lokaltherapeutika

\section{Faktorkonzentrate}

Sowohl bei FVIII- als auch FIX-Konzentraten stehen einerseits rekombinant hergestellte (r), andererseits aus Plasma hergestellte („plasma-derived“ - pd) Produkte zur Verfügung. (Siehe dazu auch den Konsensus des Wissenschaftlichen Beirats der ÖHG [6]).

Die WFH-Guidelines zum Management der Hämophilie in der gültigen Fassung geben Auskunft über die bei verschiedenen Blutungsarten anzustrebenden Plasmaspiegel von FVIII und FIX sowie über die notwendige Therapiedauer [1]. Die Autoren haben in der vorliegenden Leitlinie diese Information adaptiert, vor allem, um mehr Klarheit hinsichtlich der anzustrebenden Spitzenund Talspiegel zu erreichen (siehe Tab. 3). Die Autoren sind auch der Meinung, dass es keine ausreichende Evidenz für unterschiedliche Zielspiegel bei Hämophilie A und $B$ gibt, und haben daher diesbezüglich keine Unterscheidung getroffen. Die gegenständliche Tab. 3 wurde in drei Sitzungen nach initialem Vorschlag von vier Autoren erstellt und diskutiert, und es wurde von jedem einzelnen Autor einzeln zu jeder Indikation Zustimmung oder Ablehnung eingefordert. Am Ende wurde ein hundertprozentiger Konsensus erreicht. Die Vorschläge sind als Richtwerte zu verstehen, die ausnahmslos nicht auf randomisierten, kontrollierten Studien beruhen, sondern aus Kohortenstudie und klinischen Erfahrungswerten resultieren.

Weiters ist darauf hinzuweisen, dass bei schweren Blutungen immer normale Faktorspiegel (Talspiegel mindestens $50 \%$ ) anzustreben und entsprechende Dosierungen der Faktorkonzentrate zu verwenden sind.

Für Inhibitorpatienten stehen ein aus Plasma hergestelltes aktiviertes Prothrombinkomplexkonzentrat 
Tab. 3 Empfehlungen für FVIII/FIX-Spiegel, adaptiert nach rezenter WFH-Leitlinie. (Quelle: adaptiert nach [1]. Alle Autoren haben in allen Punkten diesen adaptierten Empfehlungen zugestimmt)

\begin{tabular}{|c|c|c|c|c|c|}
\hline \multirow[t]{2}{*}{ Blutungsart } & \multicolumn{2}{|l|}{ Zielspiegel (IU/dl) } & \multirow{2}{*}{$\begin{array}{l}\text { Dosierungs- } \\
\text { intervall (h) }\end{array}$} & \multirow{2}{*}{$\begin{array}{l}\text { Therapiedauer } \\
\text { (Tage) }\end{array}$} & \multirow[t]{2}{*}{ Kommentar } \\
\hline & $\begin{array}{l}\text { Spitzenspiegel } \\
\text { (initial) }\end{array}$ & $\begin{array}{l}\text { Talspiegel } \\
\text { (Erhaltung) }\end{array}$ & & & \\
\hline Gelenk & $40-60$ & $5-10$ & $12-24$ & 1-2, ggf. länger & $\begin{array}{l}\text { Bei großen Einblutungen sind höhere Tal- } \\
\text { spiegel anzustreben }\end{array}$ \\
\hline Muskel & $40-60$ & $5-10$ & $12-24$ & 2-3, ggf. länger & $\begin{array}{l}\text { Bedrohliche Muskelblutungen (z. B. dro- } \\
\text { hendes Kompartmentsyndrom) sind wie } \\
\text { die lliopsoas-Blutung zu behandeln }\end{array}$ \\
\hline Iliopsoas & $80-100$ & $30-60$ & 12 & $3-5$, ggf. länger & \\
\hline \multirow{2}{*}{$\begin{array}{l}\text { ZNS-Blutung bzw. andere } \\
\text { lebens- oder organbedroh- } \\
\text { liche Blutung }\end{array}$} & \multirow[t]{2}{*}{$>100$} & $50-80$ & $8-12$ & $\operatorname{Tag} 1-7$ & \multirow{2}{*}{$\begin{array}{l}\text { Dauer abhängig von Ausdehnung und } \\
\text { Resorption }\end{array}$} \\
\hline & & $30-50$ & $12-24$ & Tag 8-21 & \\
\hline Gastrointestinal & $80-100$ & $30-60$ & $12-24$ & $3-14$ & \\
\hline Niere & 50 & $15-30$ & 24 & $3-5$ & \\
\hline Tiefe RQW oder Kontusion & 50 & $5-10$ & $12-24$ & $5-7$ & \\
\hline \multirow[t]{3}{*}{ Große Eingriffe } & \multirow[t]{3}{*}{ 80-100 (prä0P) } & $60-80$ & $8-12$ & $\operatorname{Tag} 1-3$ & \\
\hline & & $40-60$ & $12-24$ & Tag 4-6 & \\
\hline & & $30-50$ & 24 & $\operatorname{Tag} 7-14$ & \\
\hline Kleine Eingriffe & 50-80 (präOP) & $30-80$ & $12-24$ & $1-5$ & Abhängig von der Art des Eingriffs \\
\hline
\end{tabular}

Tab. 4 Prophylaxe - Standarddosierung (Malmö-Protokoll). (Quelle: [1, 22])

\begin{tabular}{l|l|l|} 
& Dosis & Frequenz \\
\hline Hämophilie A & 25-40 IE/kg & Jeden 2. Tag (mindestens 3d/Woche) \\
\hline Hämophilie B & 25-40 IE/kg & Jeden 3. Tag (mindestens 2d/Woche) \\
\hline
\end{tabular}

$\left(\right.$ FEIBA $\left.^{\circledR}\right)$ und ein rekombinantes Faktor VII Konzentrat (Novoseven ${ }^{\circledast}$ ) zur Verfügung.

\section{Prophylaktische Faktorsubstitution}

Unter Prophylaxe bei Hämophilie ist die regelmäßige, von Blutungen unabhängige Substitution des fehlenden oder verminderten Blutgerinnungsfaktors mindestens einmal wöchentlich zu verstehen. Es konnte gezeigt werden, dass bei Talspiegeln von FVIII bzw. FIX von 1-2\% vor der jeweils nächsten Behandlung signifikant weniger Blutungen in Gelenke und Muskeln entstehen als bei Talspiegeln unter $1 \%[7,8]$. Bezüglich der Prophylaxedosierung gilt grundsätzlich das in Tab. 4 angeführte Schema („Malmö-Regime“), eine Individualisierung ist jedoch höchstwahrscheinlich möglich. Dafür sollten ggf. die individuelle Pharmakokinetik (Faktor-HWZ) sowie der Blutungsphänotyp herangezogen werden [9-12] - allerdings ist hier die wissenschaftliche Diskussion noch im Fluss. Auch bezüglich der Parameter für eine erfolgreiche Prophylaxe besteht derzeit international keine Einigkeit.

\section{Therapeutische Faktorsubstitution (Bedarfsbehandlung)}

Im Gegensatz zur Prophylaxe versteht man unter der therapeutischen Faktorsubstitution die Verabreichung des fehlenden oder verminderten Blutgerinnungsfaktors im
Blutungsfall (d. h. bei Bedarf - „on demand“). Bei schwerer Hämophilie und kleineren Blutungen (z. B. beginnende Gelenksblutung oder Weichteilhämatom nicht Hb-wirksam) beträgt die Einzeldosierung meist 20-30 IE/ $\mathrm{kg}$, bei größeren Blutungen (in Gelenke und/oder Muskulatur oder Hb-wirksam) 40-60 IE/kg. Um einen optimalen Effekt zu erzielen, sollte die Substitution schon bei den ersten Anzeichen einer Blutung begonnen und dann alle 8-12 bzw. $24 \mathrm{~h}$ weiter gegeben werden, je nach klinischer Situation und Faktorspiegel. Bei Gelenksblutungen kann in ca. 70-80\% mit einer einmaligen Gabe eines Faktorkonzentrates ein Blutungsstillstand und damit eine effektive Therapie erreicht werden.

\section{Heimtherapie}

Unter Heimtherapie versteht man die intravenöse Verabreichung von Faktorkonzentrat für Prophylaxe und Therapie zu Hause durch den Patienten selbst oder eine Betreuungsperson. Im Sinne einer alltagsgerechten und im Bedarfsfall möglichst schnellen Behandlung muss das Ziel sein, die Behandlung mittelfristig zu Hause, am Arbeitsplatz oder auf Reisen durchführen zu können. Die praktische Organisation und Durchführung der Heimtherapie soll an die individuellen Gegebenheiten des Patienten und seines sozialen Umfelds (Eltern, Familie, Betreuungspersonen) angepasst werden. Dies kann initial durch den Einsatz mobiler Krankenschwestern und im weiteren durch die Information und Schulung der Eltern, später der betroffenen Kinder, Jugendlichen und Erwachsenen, erreicht werden. Niedergelassene Allgemeinmediziner, Kinderfachärzte und periphere Kinderabteilungen können ebenfalls in die Schulung involviert werden [12]. 


\section{Empfehlungen Faktorkonzentrate}

- Die Empfehlungen hinsichtlich Zielspiegel und Therapiedauer bei verschiedenen Blutungsarten sind in Tab. 3 zusammenfassend dargestellt.

- Bei schweren Blutungen sollten besonders in den ersten Tagen immer normale Faktorspiegel (Talspiegel 50-80\%) angestrebt werden.

- Bei Operationen, schweren Blutungen oder schweren Traumen soll die Therapie durch regelmäßige Bestimmungen des Faktorspiegels überwacht werden (z. B. Talspiegel 1x täglich vor der morgendlichen Gabe des Faktorkonzentrates).

- Speziell bei der Hämophilie B ist die niedrigere Wiederfindungsrate (Recovery) von Faktor IX zu beachten.

- Die prophylaktische Substitutionstherapie und die Bedarfsbehandlung sollten durch den Patienten selbst oder seine Betreuungspersonen zu Hause (Heimtherapie) erfolgen.

\section{Desmopressin (DDAVP)}

DDAVP ist ein synthetisches Analogon des antidiuretischen Hormons Vasopressin und kann bei Patienten mit leichter oder mittelschwerer Hämophilie A und Konduktorinnen der Hämophilie A eingesetzt werden. Bei der Hämophilie B ist es nicht wirksam. DDAVP kann intravenös, intranasal oder subkutan verabreicht werden. Bei akuten schweren Blutungen und Epistaxis sollten intranasale Gaben aufgrund des unsicheren Ansprechens vermieden werden. Bei Hämophilie A führt Desmopressin zu einem Anstieg des FVIII und des von-Willebrand-Faktors um das 3- bis 6-Fache (1). Die Dosierungen sind in Tab. 5 angegeben. Eine „Testinfusion“ vor einem geplanten Eingriff wird empfohlen. Dabei wird der FVIII vor und nach der Infusion gemessen, nach $60 \mathrm{~min}$ ist nach intravenöser Gabe der höchste Wirkspiegel erreicht. Dies erlaubt eine Einschätzung des zu erwartenden FVIIISpiegels nach der Infusion und damit einen Rückschluss auf die blutungshemmende Wirkung des Medikamentes in einer bestimmten Situation. Zusätzlich besteht eine direkte Wirkung auf die Thrombozytenfunktion. Es ist wichtig, beim Desmopressineinsatz das Risiko einer Wasserintoxikation zu beachten. Für Kinder unter zwei Jahren und Patienten mit Anfallsleiden ist Desmopressin grundsätzlich nicht empfohlen. Kinder haben ein besonderes Risiko für Krampfanfälle aufgrund eines durch Wasserretention bedingten Hirnödems. Bei Kindern zwischen zwei und sechs Jahren kann Desmopressin mit Vorsicht und unter strenger Observanz hinsichtlich Trinkmenge und Ausscheidung angewandt werden [1]. Weiters besteht eine Kontraindikation bei Patienten mit instabiler Angina pectoris oder bekannter Atherosklerose [13]. Es wird auf die Beachtung der in der Fachinformation aufgeführten Kontraindikationen und Vorsichtsmaßnahmen hingewiesen.
Tab. 5 Dosisempfehlung für Desmopressin bei Patienten mit Hämophilie A. (Quelle: AWMF-Leitlinie „Thrombozytopathien - Therapie“, AWMF-Register Nr. 086-004, Klasse: S2K, erhältlich unter http://www.awmf.org/leitlinien/detail/ II/086-004.html)

\begin{tabular}{|c|c|c|c|}
\hline \multirow[t]{2}{*}{ Patienten } & \multicolumn{2}{|l|}{ Therapie } & \multirow{2}{*}{$\begin{array}{l}\text { Präoperative Prophylax } \\
\text { Intravenös } 60 \text { min vor } \\
\text { dem Eingriff }\end{array}$} \\
\hline & Intranasal & $\begin{array}{l}\text { Intravenös/ } \\
\text { subcutan }\end{array}$ & \\
\hline$\leq 12$ Jahre & $\begin{array}{l}150 \mu \mathrm{g}(1 \\
\text { Sprühstoß) }\end{array}$ & \multirow[t]{4}{*}{$\begin{array}{l}0,3-0,4 \mu \mathrm{g} / \mathrm{kg} \\
\text { über } 30 \mathrm{~min}\end{array}$} & \multirow[t]{4}{*}{$\begin{array}{l}0,3-0,4 \mu \mathrm{g} / \mathrm{kg} \text { über } \\
30 \mathrm{~min}\end{array}$} \\
\hline$>12$ Jahre & $\begin{array}{l}300 \mu \mathrm{g}(2 \\
\text { Sprühstöße) }\end{array}$ & & \\
\hline$\leq 50 \mathrm{~kg}$ & $\begin{array}{l}150 \mu \mathrm{g}(1 \\
\text { Sprühstoß) }\end{array}$ & & \\
\hline$>50 \mathrm{~kg}$ & $\begin{array}{l}300 \mu \mathrm{g}(2 \\
\text { Sprühstöße) }\end{array}$ & & \\
\hline
\end{tabular}

Eine Kombination von Desmopressin mit Tranexamsäure ist sinnvoll, insbesondere bei Schleimhautblutungen oder Eingriffen an Schleimhäuten. Desmopressin soll aufgrund der zu erwartenden Tachyphylaxie nur wenige Tage verwendet werden. Falls zu diesem Zeitpunkt noch keine Blutstillung erreicht werden konnte, müssen andere Maßnahmen ergriffen werden.

\section{Antifibrinolytika (Tranexamsäure)}

Bei Hämophiliepatienten kann bei bestimmten Blutungen und Eingriffen adjuvant ein Antifibrinolytikum (Tranexamsäure) verabreicht werden. Antifibrinolytika sind besonders effektiv bei Schleimhautblutungen wie z. B. Epistaxis; die Anwendung von Tranexamsäure alleine hat jedoch keinen präventiven Effekt bezüglich Gelenksblutungen [1, 14]. Tranexamsäure kann lokal, per os oder intravenös eingesetzt werden. Üblicherweise werden nach einmaliger Bolusgabe 10-15 mg/kg KG i.v. $3 \mathrm{x}$ tgl. oder 20-25 mg/kg KG p.o. 3-4x tgl. verabreicht. Eine Dosisreduktion bei eingeschränkter Nierenfunktion ist notwendig.

Besonders nützlich ist Tranexamsäure bei zahnärztlichen Eingriffen, sie kann auch zur Stillung von Blutungen rund um den Zahnwechsel verwendet werden (s. den Abschnitt zum Vorgehen bei Operationen und Zahnextraktionen weiter unten) $[15,16]$. Bei Blutungen im Mundschleimhautbereich sind Mundspülungen mit anschließendem Verschlucken der mit isotoner Kochsalzlösung verdünnten äquivalenten oralen Dosen sinnvoll. Die Therapiedauer beträgt fünf (bis zehn) Tage.

Tranexamsäure kann in Kombination mit Faktorkonzentrat in Standarddosen verabreicht werden [1, 17]. Falls eine Therapie sowohl mit Tranexamsäure als auch mit aktivierten Prothrombinkomplex-Konzentraten (APCC, FEIBA ${ }^{\circledR}$ ) erforderlich ist, sollten jeweils zwölf Stunden zwischen den Applikationen liegen $[1,18]$. Eine Erhöhung des Thromboembolierisikos bei Gabe von Tranexamsäure in Kombination mit rekombinantem Faktor VIIa ist nicht bekannt $[1,19]$. 
Bei Hämaturie/Nierenblutungen sollten Antifibrinolytika aufgrund der Gefahr der Bildung von abflussbehindernden Blutgerinnseln nicht oder nur unter konsequenter Ultraschallkontrolle eingesetzt werden.

\section{Analgetika}

Akute und chronische Schmerzen sind bei der Hämophilie häufig. Bei der Schmerztherapie ist auf die unterschiedlichen Schmerzursachen Bedacht zu nehmen [1]. Schmerzen beim Legen eines venösen Zugangs können durch Lokalanästhetika gelindert werden. Für Schmerzen, die durch Gelenks- oder Muskelblutungen entstehen, sollten neben einer möglichst frühzeitigen Gerinnungsfaktor-Substitution auch adäquate analgetische Maßnahmen getroffen werden.

Tabelle 6 zeigt ein analgetisches Stufenschema für Hämophiliepatienten. Dabei ist zu beachten, dass bei Kindern keine COX-2-Hemmer verwendet werden. Generell sind bei Patienten mit Hämophilie intramuskuläre Injektionen in den Gesäßmuskel zu vermeiden.

Die postoperative Schmerztherapie soll in Absprache mit dem Anästhesisten erfolgen und kann z. B. mit parenteralen Opioiden beginnen und bei nachlassendem Schmerz auf COX-2 Hemmer oder Paracetamol umgestellt werden [1].

Die Therapie der chronisch-hämophilen Arthropathie umfasst funktionelles Training und adäquate Analgesie gemäß Tab. 6, wobei COX-2-Hemmer hier eine größere Rolle spielen, während andere NSAR in der Schmerztherapie vermieden werden sollten. Patienten mit persistierenden Schmerzen sollten in Zusammenarbeit mit spezialisierten Schmerztherapeuten behandelt werden [1]. Bei verzögert auf Substitution ansprechenden Gelenksblutungen und Hinweisen auf subakute bis chronische Synovitis kann im Einzelfall eine analgetisch/ antiphlogistische Therapie mit Ibuprofen oder Naproxen für begrenzte Zeit und unter ausreichender Faktorsubstitution unter Abwägung von Vor-und Nachteilen in Erwägung gezogen werden.

Die Anwendung von Kühlung mittels Eisbeuteln und dgl. bei Gelenksblutungen wird subjektiv als angenehm empfunden - die Evidenz für diese Maßnahme fehlt weitgehend. Kühlung soll nach subjektivem Nutzen durchgeführt werden.

\section{Lokaltherapeutika und unspezifische Maßnahmen}

Unspezifische Maßnahmen zur Blutstillung und lokale Hämostyptika können die Behandlung von akuten Blutungen sinnvoll ergänzen. Mit Kaolin, Thrombin und Tranexamsäure getränkte Wundauflagen, wie Quikclot Combat Gaze $^{\circledast}$ oder Hematrix ${ }^{\circledast}$ führen zu einer rascheren Blutstillung bei offenen, stark blutenden Verletzungen im Vergleich zu Standardverbänden. Bewährt hat sich bei Hämophilen die lokale Anwendung von mit Tranexamsäure-Injektionslösung getränkten Gazen.
Tab. 6 Analgetisches Stufenschema für Hämophiliepatienten. (Quelle: adaptiert nach [1])

\begin{tabular}{|l|l|}
\hline Stufe & Verwendete Substanzen \\
\hline 1 & Paracetamol \\
\hline 2 & $\begin{array}{l}\text { COX-2-Hemmer (cave Hypertonie und Nierenfunktion; nicht } \\
\text { bei Kindern!) Paracetamol plus Codein ODER Paracetamol plus } \\
\text { Tramadol }\end{array}$ \\
\hline 3 & $\begin{array}{l}\text { Opioide (retardierte Präparate als Basismedikation plus schnell } \\
\text { wirksame Präparate als Zusatzoption; wenn letztere mehr als } \\
\text { viermal täglich verwendet werden müssen, sollte die Basismedi- } \\
\text { kation gesteigert werden. Wenn aus irgendeinem Grund bei einem } \\
\text { Patienten, der hochdosierte Analgetika erhalten und vertragen } \\
\text { hat, die Therapie unterbrochen wird, sollte ein Wiedereinstieg in } \\
\text { niedrigerer Dosis erfolgen) }\end{array}$ \\
\hline
\end{tabular}

Tab. 7 Weitere Maßnahmen bei Blutungen. (Quelle: Autoren)

Die Entscheidung zur Transfusion von Erythrozytenkonzentraten soll für jeden Patienten individuell auf Basis des klinischen Gesamtbildes erfolgen. Liegt der Hämoglobinwert < 7-8 g/dl soll eine Transfusion ernsthaft überlegt werden. Bei Patienten mit koronaren, kardiovaskulären oder kardio-I pulmonalen Beeinträchtigungen ist der Schwellenwert für die Transfusionsentscheidung bei einem Hämoglobinwert von 9,5-10 g/dl anzusetzen

Blutenden Patienten mit einer Blutplättchenzahl $<50.000 / \mu l$ sollten Thrombozytenkonzentrate transfundiert werden. Bei Blutungen in kritische Organe (z. B. Zentralnervensystem oder Auge) soll bereits ab Thrombozytenwerten von $<100.000 / \mu l$ transfundiert werden

Vermeidung von Thrombozytgenaggregationshemmern wie ASS oder NSAR Lokaltherapie: Kompression, Fibrinkleber, lokale Hämostyptika

Antifibrinolytika: Tranexamsäure lokal, enteral, parenteral auch kombiniert

In Tab. 7 sind weitere allgemeine Maßnahmen bei Blutungen zusammengefasst.

Empfehlungen Desmopressin, Fibrinolysehemmer und Schmerztherapie

- Desmopressin ist eine wirksame Alternative bei leichter, ev. mittelschwerer Hämophilie A und Konduktorinnen der Hämophilie A mit verminderten FVIII-Spiegeln bei Blutungen und invasiven Eingriffen (Level 3).

- Bei allen Hämophiliepatienten sollte adjuvant ein Fibrinolysehemmer (Tranexamsäure) bei Schleimhautblutungen (Level 2) und Zahnextraktionen (Level 4) verabreicht werden.

- Die Schmerztherapie sollten bei Hämophiliepatienten nach einem analgetischen Stufenschema (Tab. 6) durchgeführt werden, ggf. auch unter Beiziehung spezialisierter Schmerztherapeuten. Von Medikamenten mit Thrombozytenaggregations-hemmenden Eigenschaften (NSAR) ist abzuraten. 


\section{Spezielle Aspekte der Prophylaxe und Therapie bei Kindern mit schwerer Hämophilie}

Die vielfältigen Aufgaben in der Betreuung von Kindern mit Hämophilie von der Aufklärung der Eltern bis zum Angebot psychotherapeutischer Unterstützung sind in Tab. 8 aufgelistet. Die wichtigste Maßnahme ist die Substitution mit Faktorkonzentraten, um Blutungen zu verhindern oder zu therapieren.

Ein Beginn einer prophylaktischen Faktorsubstitution in der Behandlung im ersten bis zweiten Lebensjahr bei schweren Hämophilen (wenn möglich jedenfalls vor dem ersten oder zweiten dokumentierten Blutungsereignis) führt zu weniger Gelenksblutungen, weniger konsekutiven Arthropathien und zu einer deutlich höheren Lebensqualität [20, 21].

Als primäre Prophylaxe ist die Faktorsubstitution vor Auftreten einer zweiten Gelenksblutung definiert [1]. Sie wird individuell begonnen, meist zwischen dem 6 . und 18. Lebensmonat $[22,23]$. Als sekundäre Prophylaxe ist der Beginn der Substitution von Faktorkonzentraten erst nach dem Auftreten von mindestens zwei Gelenksblutungen, aber noch ohne Nachweis von Gelenksschäden definiert. Nach den ersten Gelenksblutungen können bereits irreversible Schädigungen von Gelenken und insbesondere von Knorpelgewebe entstehen [22].

Wenngleich es Argumente für einen frühen Beginn der primären Prophylaxe gibt, können dem auch Hindernisse entgegenstehen. Solche Hindernisse für eine frühe Prophylaxe sind z. B. eine schlechte Venensituation, organisatorische Herausforderungen der regelmäßigen Faktorgabe beim Kleinkind oder die (initial) fehlende Akzeptanz der Prophylaxe durch die Patientenfamilie. Ein Beginn der Prophylaxe vor Erreichen des 1. Lebensjahres führt weder zu einer Senkung noch zu einer Erhöhung des Hemmkörperrisikos.

Unter Prophylaxe ist die Freiheit von schweren Blutungen anzustreben. Wenn unter laufender Prophylaxe Gelenksblutungen auftreten, so muss zuallererst an das Auftreten eines Hemmkörpers gegen den verabreichten Faktor gedacht werden. Bei negativem Hemmkörpertest ist generell eine Steigerung der Häufigkeit und/oder der Dosis vorzunehmen. In Abhängigkeit von Lokalisation und Schweregrad der Blutung werden unterschiedliche Regime empfohlen. Dabei können Dosierungen von bis zu 80 IE/kg verwendet werden [20]. In der Regel werden jedoch niedrigere Dosierungen verabreicht [12].

Die Impfempfehlungen für Kinder mit Hämophilie sind in Tab. 8 angeführt. Für Patienten mit Hämophilie besteht bei intramuskulären Impfungen ein erhöhtes Risiko für Muskelblutungen. Es wird daher empfohlen, Impfungen prinzipiell subkutan zu verabreichen. Für einige Impfstoffe, wie z. B. Haemophilus influenzae und Hepatitis B gibt es bereits Daten über eine vergleichbare Impfantwort nach subkutaner und intramuskulärer Impfung [24]. Extrapolierend wird auf Basis bisher vorhandener Daten davon ausgegangen, dass auch bei den anderen Impfungen eine subkutane Gabe zu vergleichbaren Impfantworten führt [25].
Tab. 8 Aufgaben bei der Betreuung von Kindern mit Hämophilie. (Quelle: Autoren)

Aufklärung der Eltern über: Erkrankung, Vererbung, Erkennen und Management von Blutungen, Komplikationen und Prognose

Ausgabe von Informationsmaterial

Faktorauswahl (rekombinantes oder aus Plasma hergestelltes Produkt) und Organisation der Faktorbestellung

Ausstellen eines Patientenausweises

Schulung der Eltern (Faktorzubereitung, Injektion)

Impfempfehlung an Kinderärzte (alle empfohlenen Impfungen plus Hepatitis A)

Allgemeine Empfehlungen, wie Zahnhygiene, Unfallprophylaxe

Sportempfehlungen (erlaubt: z. B. Gymnastik, Laufen, Schwimmen, Radfahren, Skifahren und Skaten mit Helm; nicht empfohlen bzw nur mit besonderer Vorsicht (speziell angepasste Prophylaxe, Sicherungsmass-nahmen, regelmässige Kontrollen): Extrem- und Hochleistungssport, Kontaktsportarten, Stopp-and-go-Sportarten, wozu auch die meisten Teamsportarten zählen, Kampf-und Sprungsport)

Information der außerfamiliären Betreuungspersonen und Einrichtungen

Bestätigung zur Vorlage bei Krankenkassen und Ämtern

Eventuell psychotherapeutische Unterstützung der Familie

\section{Empfehlungen zur Therapie bei Kindern mit} schwerer Hämophilie

- Kinder mit schwerer Hämophilie sollen eine primäre Prophylaxe erhalten (Level 1)

- Mit einer prophylaktischen Faktorsubstitution sollte spätestens nach der ersten Gelenksblutung begonnen werden.

- Aufgrund eines möglicherweise höheren Hemmkörperrisikos sollte, soweit klinisch vertretbar, der Beginn einer Prophylaxe so gewählt werden, dass ein Abstand von 3-6 Wochen zwischen Immunisierungen nach Impfplan und/ oder Blutungsproblemen eingehalten wird.

- Die Dosierung soll initial 20-40 IE/kg ein- bis zweimal wöchentlich betragen. Bei Auftreten von Blutungen sollte nicht die Einzeldosis, sondern zunächst die Frequenz der Verabreichung gesteigert werden.

- Die Dosierung kann im Weiteren, idealerweise auch abhängig von individuellen Parametern (Faktor-Restaktivität, pharmakokinetischen Parametern wie Recovery und Halbwertszeit), angepasst werden.

- Bei mittelschwerer Hämophilie hängt die Prophylaxe vom klinischen Phänotyp ab.

\section{Venöse Zugänge}

Der Zugang über eine periphere Vene ist auch bei Kleinkindern immer die erste Wahl, da er keinen chirurgischen Eingriff erfordert und die niedrigste Komplikationsrate aufweist. Wenn dies nicht möglich ist, bestehen zwei Alternativen: 
- Port-a-cath-System: Hier handelt es sich um die operative Implantation einer subkutanen Kammer mit einer durch die Haut anstechbaren Membran, die Anschluss an das zentrale Venensystem hat. Port-acaths werden zumeist über dem Musculus pectoralis fixiert, der ein gutes Widerlager darstellt. Vorteil: Sofort für Faktorgaben verwendbar. Nachteile bzw. mögliche Komplikationen: Infektion, tiefe Venenthrombose, mechanische Dysfunktion (Okklusion, Membrandefekt, Diskonnektion), Dislokation, Hautnekrose.

- Arteriovenöser Shunt: Hier handelt es sich um eine chirurgisch hergestellte Anastomose zwischen einer Arterie und einer oberflächlichen Vene an der oberen Extremität (Gracz-Shunt: A. brachialis und Unterarmvene [V. cephalica, V. basilica, V. mediana cubiti oder V. perforans]; Brescia-Cimino-Shunt: A. radialis und V. cephalica). Vorteil: Leichte Punktierbarkeit nach Venenstauung analog zu peripherer Venenpunktion; Komplikationsrate wahrscheinlich geringer als bei Port-a-cath.

- Nachteile bzw. mögliche Komplikationen: Okklusion, Phlebitis, Schwellung des operierten Arms (Risiko beim Gracz-Shunt aufgrund niedriger Durchflussrate geringer als beim Brescia-Cimino-Shunt), Verletzungsrisiko; erst nach „Reifen“ des Shunts verwendbar (nach ca. zwei bis vier Wochen); Wachstumsstörung des Armes.

Es liegen keine direkt vergleichenden Studien zwischen Port-a-cath-System und arteriovenösem Shunt vor. Beide können je nach Situation des Patienten empfohlen werden. Die Eltern/der Patient sollten über beide Möglichkeiten informiert werden und eine gemeinsame Entscheidung mit den Betreuern treffen.

Empfehlungen venöse Zugänge

- Erste Wahl ist der periphere venöse Zugang.

- Ist dies nicht möglich, so stehen ein Port-a-cathSystem oder ein arteriovenöser Shunt als Alternativen zur Verfügung, wobei zwischen diesen beiden Möglichkeiten je nach Situation des Patienten entschieden werden soll (Level 2).

\section{Spezielle Aspekte der Therapie und Prophylaxe} bei Erwachsenen mit schwerer Hämophilie

Therapie und Prophylaxe bei Erwachsenen sind im Wesentlichen so $\mathrm{zu}$ handhaben wie bei Kindern und Jugendlichen. Laut WFH wird eine Fortführung der primären prophylaktischen Verabreichung von Faktorkonzentraten bzw. die Einleitung einer sekundären Prophylaxe bei Hämophilie auch für Erwachsene emp- fohlen, weil der Schweregrad der Hämophilie auch im Erwachsenenalter fortbesteht, das Risiko spontan auftretender und traumatischer Blutungen bestehen bleibt und die aus der Prophylaxe resultierenden Vorteile in allen Altersgruppen vorhanden sind. Eine sekundäre Prophylaxe kann zwar die hämophile Arthropathie nicht verhindern, aber ihren Schweregrad vermindern [20, 26-32]. Bei älteren Erwachsenen mit schwerer Hämophilie und hämophiler Arthropathie wird oft noch eine Therapie bei Bedarf durchgeführt, und eine Reihe dieser Patienten wünschen gar keine Prophylaxe.

In besonderen Situationen, z. B. nach orthopädischen Eingriffen oder nach schweren oder immer wiederkehrenden Blutungen in ein Gelenk, ist eine zeitlich begrenzte Prophylaxe (über Wochen oder Monate) in der Zeit der Wiederherstellung, oft verbunden mit Physiotherapie, eine sehr gute Alternative zu einer reinen Bedarfstherapie oder einer langfristigen Prophylaxe. Auf spezielle klinische Situationen, wie z. B. hoch betagte Patienten oder Patienten mit Komorbiditäten wie Vorhofflimmern oder st.p. koronarer Stentimplantation, wird hier nicht eingegangen, da gerade auf diesen Gebieten derzeit neue internationale Leitlinien erarbeitet werden.

\section{Empfehlungen zur Therapie bei Erwachsenen mit schwerer Hämophilie}

- Auch für erwachsene Patienten mit schwerer Hämophilie wird eine prophylaktische Verabreichung von Faktorkonzentrat routinemäßig empfohlen. Dies kann sowohl die Weiterführung einer schon in der Kindheit begonnenen Prophylaxe als auch die Neu- oder Wiedereinführung einer Prophylaxe im Erwachsenenalter bei entsprechender Indikation (Blutungen) bedeuten.

\section{Mittelschwere und leichte Hämophilie}

Patienten mit mittelschwerer Hämophilie und Blutungen sollten wie Patienten mit schwerer Hämophilie behandelt werden. In allen anderen Fällen ist eine Therapie bei Bedarf indiziert.

Für Patienten mit leichter Hämophilie A kann als Alternative zur Steigerung der Faktor-VIII-Aktivität eine Therapie mit Desmopressin (siehe Kapitel medikamentöse Therapien) bei Eingriffen, traumatischen Blutungen, Hämaturie und Epistaxis verwendet werden. Auf Aspekte der Desmopressin-Therapie wird bereits in einem vorherigen Kapitel eingegangen. Bei Hämophilie B ist Desmopressin nicht wirksam. Bei großen Operationen oder schweren Blutungen ist auch bei Patienten mit leichter Hämophilie eine Substitution mit Faktorkonzentraten anzuwenden. Die Zielspiegel sind in Tab. 3 angeführt. 
Empfehlungen für Patienten mit mittelschwerer und leichter Hämophilie

- Patienten mit mittelschwerer Hämophilie und dem klinischen Bild einer schweren Form sollen prophylaktisch behandelt werden, ansonsten erfolgt eine Behandlung bei Bedarf.

- Für Patienten mit mittelschwerer oder leichter Hämophilie A kommt als Alternative zur Steigerung der Faktor-VIII-Aktivität eine Therapie mit Desmopressin unter Beachtung der Kontraindikationen in Frage (Level 3). Bei schweren Blutungen oder großen Operationen ist, besonders in den ersten Tagen, eine Substitution mit Faktorkonzentraten in Erwägung zu ziehen.

- Für Patienten mit Hämophilie B werden FaktorIX-Konzentrate zur Therapie und Prophylaxe (z. B. perioperativ) eingesetzt.

- Die erforderlichen Spiegel aus Tab. 3 gelten auch für Patienten mit mittelschwerer und leichter Hämophilie.

\section{Spezielle Blutungen bei Kindern und Erwachsenen}

\section{Nierenblutungen}

Hämophilie-bedingte Hämaturie sollte mit Bettruhe und forcierter Hydrierung über $48 \mathrm{~h}$ behandelt werden. Während der intensiven Hydrierung sollte die Gabe von Desmopressin vermieden werden [1,33]. Eine Substitution mit Faktorkonzentraten kann erfolgen, Dosierungsangaben sind Tab. $3 \mathrm{zu}$ entnehmen. Wenn Schmerzen bzw. Makrohämaturie über 12 bis $24 \mathrm{~h}$ persistieren, sollte nach Thromben, Obstruktion und anderen Ursachen in den ableitenden Harnwegen gefahndet werden [1, 33, 34], ebenso bei rezidivierenden Episoden einer Hämaturie. Bei Harnverhaltung bei vorangegangener Makrohämaturie ist eine unverzügliche Abklärung notwendig. Antifibrinolytika sollen wegen der Gefahr einer Obstruktion mit Harnrückstau nicht oder nur in Ausnahmefällen unter strenger sonographischer Kontrolle verwendet werden [33].

\section{Blutungen der Mundschleimhaut}

Die Therapie von Blutungen der Mundschleimhaut erfolgt einerseits durch Lokalmaßnahmen wie direkten Druck auf die Blutungsstelle mit einem feuchten Tupfer über ca. 15 min, ggf. Nähte, lokale Applikation von Tranexamsäure oder Hämostyptika (siehe entsprechende Kapitel), andererseits durch die parenterale oder enterale Gabe von Tranexamsäure (Cyklokapron ${ }^{\circledR}$ ). Bei schlechter Mundhygiene sind Mundhygienemaßnahmen durchzuführen, und Antibiotika können ev. hilfreich sein. Eine Faktorsubstitution kann bei persistierender Blutung und bei notwendigem operativem Eingriff oder professio- nell durchgeführter Mundhygiene erforderlich sein [1]. Erwachsene Patienten sollten angewiesen werden, das Verschlucken von Blut zu vermeiden [1].

Die möglichst frühe Beiziehung eines Zahnarztes oder Kieferchirurgen ist wichtig, um die Blutungsquelle zu identifizieren. Die häufigsten Ursachen für Blutungen sind Zahnwechsel und -extraktion, Zahnfleischblutung aufgrund schlechter Mundhygiene und Trauma [1].

\section{Epistaxis}

Epistaxis kann nicht selten durch Befeuchtung der Raumluft und/oder Verwendung von Kochsalz-hältigen Nasensprays verhindert werden [1].

Bei Epistaxis hat sich eine frühzeitige Tamponade mit Tranexamsäure-getränkter Gaze zu Hause bewährt. Auf Abrinnspuren im Pharynx ist immer zu achten. Die systemische Anwendung von Antifibrinolytika ist empfohlen.

Der Kopf des Patienten sollte nach vorne gebeugt sein, um das Verschlucken von Blut zu vermeiden und das vorsichtige Ausschneuzen von Koageln zu ermöglichen. Mit einem in Eiswasser getränkten Gazetupfer sollte zehn bis 20 min lang fester Druck auf den vorderen, knorpeligen Teil der Nase ausgeübt werden [1]. Eine Faktorsubstitution ist oft nicht notwendig, außer bei schweren oder rezidivierenden Blutungen $[1,35,36]$.

Antihistaminika und Schleimhaut-abschwellende Mittel können bei Blutungen im Zusammenhang mit Allergien, Infektionen des oberen Respirationstrakts und saisonalen Veränderungen hilfreich sein [1].

Bei lang anhaltenden oder häufig rezidivierenden Blutungen sollte auf eine mögliche Anämie geachtet und diese ggf. adäquat behandelt werden. Weiters ist in diesem Fall ein HNO-Arzt zu konsultieren [1]. Die lokale (getränkter Tupfer) oder systemische Anwendung von Antifibrinolytika ist hilfreich [1]. Bei Kindern wird eine systemische Anwendung empfohlen.

\section{Vorgehen bei Operationen und Zahnextraktionen}

Bei Operationen, anderen invasiven Eingriffen und Zahnextraktionen sind Faktorkonzentrate zur Vermeidung verstärkter Blutungsneigung hoch wirksam. Jedoch sind weder Dosierung noch Dosisintervalle der Faktorgaben rund um chirurgische Eingriffe wissenschaftlich untermauert. Die derzeitigen Behandlungsprinzipien beruhen daher in erster Linie auf klinischer Erfahrung. Zu den erforderlichen Faktor-Plasmaspiegeln vor Eingriffen siehe Tab. 3. Eine stringente und allgemein akzeptierte Definition eines „großen“ bzw. „kleinen“ Eingriffs bei Hämophilen existiert nicht. Versuche, eine solche Definition nach der Dauer der fortgesetzten notwendigen Faktorverabreichung [37] oder nach Blutungsrisiko bzw. Blutverlust [38] festzulegen, erscheinen als nicht ausreichend. Vielmehr beruhen die Entscheidung über das Blutungsrisiko bei einem Eingriff und die sich daraus ableitenden Vorsichtsmaßnahmen (Substitution) 
bei Hämophiliepatienten weitgehend auf klinischer Erfahrung.

Die Verabreichung von Desmopressin bei leichter Hämophilie A vor Operationen ist etabliert; das Ansprechen auf Desmopressin sollte vorher ausgetestet werden (siehe auch den Abschnitt zu Desmopressin weiter oben). Die Kombination von Desmopressin mit Antifibrinolytika sollte auf die Anwendung bei Schleimhautblutungen beschränkt bleiben [13]. Tranexamsäure soll bei Eingriffen im Schleimhautbereich immer zusätzlich verabreicht werden (s. auch den Abschnitt zu Antifibrinolytika weiter oben).

Eine kontinuierliche Infusion von Faktorkonzentraten ist in Österreich nicht Standard und wird daher hier nicht näher behandelt.

Generell ist es zu empfehlen, dass der Hämophiliepatient vor jedem geplanten operativen Eingriff seinen Hämophiliebehandler kontaktiert. Der Behandler sollte zehn bis 14 Tage präoperativ einen Therapieplan erstellen. Präoperativ und bei klinischem Verdacht jederzeit sollte eine Inhibitortestung erfolgen. Laborkontrollen von FVIII oder FIX sollten vor und nach Verabreichung der präoperativen „loading dose“, danach einmal täglich als Talspiegel (z. B. vor der morgendlichen Faktorgabe) erfolgen. Die „loading dose“ sollte zeitnah (nicht länger als 2-3 h, besser ca. 15-30 min vor Beginn des Eingriffes) vor der Operation verabreicht werden. Wenn kein Inhibitor vorhanden ist, muss nach ausreichender Substitution mit dem Beginn der Operation nicht auf den Laborwert der jeweiligen Plasmafaktorkonzentration gewartet werden. Falls dieser sich jedoch als zu niedrig erweist, ist intra- oder postoperativ eine entsprechende weitere Dosis zu substituieren. Am Operationstag sollte in Abständen von acht bis zwölf Stunden substituiert werden. Entscheidend ist in jedem Fall die Einhaltung fixer, vorgegebener Substitutionsintervalle. Die Adaptierung der Dosis sollte nach dem vor einer Faktorverabreichung abgenommenen Talspiegel erfolgen. Es ist jedoch darauf zu achten, dass die regelmäßige Substitution durch Warten auf das Laborergebnis keinesfalls verzögert wird.

Patienten mit milder Hämophilie sowie jene, die zum ersten Mal große Mengen an Gerinnungsfaktoren erhalten, sollten vier bis zwölf Wochen postoperativ auf Vorliegen eines Inhibitors kontrolliert werden[1].

Für Zahnextraktionen wird eine Faktorsubstitution bis zu einem Spiegel von mindestens $50 \%$ empfohlen [39]. Eine antifibrinolytische Therapie wird für sieben Tage empfohlen (Level 2). Eine zusätzliche Blutstillung mit Fibrinkleber soll in Erwägung gezogen werden (Level 3) $[15,16,39-41]$.

Bei weiblichen Verwandten ersten Grades (Mutter, Schwester, Tochter) von Hämophiliepatienten sollte vor einer Geburt bzw. vor einem ersten operativen Eingriff eine Gerinnungsfaktorkontrolle erfolgen, da Konduktorinnen in Abhängigkeit von der Höhe des Gerinnungsfaktors bei Operationen eine erhöhte Blutungsneigung haben können [1]. Therapievorschläge für Konduktorinnen werden in diesen Leitlinien gesondert weiter hinten abgehandelt.
Bei Hämophiliepatienten sieht die Leitliniengruppe generell keine Indikation für eine postoperative medikamentöse Thromboseprophylaxe.

\section{Empfehlungen für Dosisberechnungen Empfehlungen für Tal- und Spitzenspiegel von FVIII bzw FIX}

\section{Hämophilie A ohne Inhibitor}

1 IE FVIII/kg KG i.v. erhöht den Plasma-FVIIISpiegel um ca. 2 IE/dl. Die HWZ von FVIII liegt bei acht bis zwölf Stunden. 15-30 min nach Verabreichung des Faktorkonzentrates sollte der FVIIISpiegel kontrolliert werden [42].

Formel zur Dosisberechnung:

$\mathrm{KG}(\mathrm{kg}) \times$ gewünschter Faktoranstieg $(\mathrm{IE} / \mathrm{dl}) \times 0,5$ Folgedosen sollten auf Basis von Talspiegeln und der individuellen Recovery und Halbwertszeit angepasst werden.

Beispiel:

Um bei einem Patienten mit schwerer Hämophilie einen Spiegel von $100 \%$ FVIII-Aktivität zu erzielen, sind ca. $50 \mathrm{IE} / \mathrm{kg}$ KG notwendig. Bei einer HWZ von ca. 12h ist der Spiegel nach einem halben Tag auf etwa $50 \%$ abgefallen, die weiteren Dosierungen sollten in 8- bis 12-stündlichen Intervallen gegeben werden. Regelmäßige Messungen (z. B. einmal täglich) des Talspiegels sollten durchgeführt werden.

\section{Hämophilie B ohne Inhibitor}

Bei Hämophilie B ist die Recovery etwa halb so hoch wie bei FVIII. Um den FIX-Spiegel um $1 \%$ anzuheben, ist die Gabe von 1 IE/ $\mathrm{kg}$ KG erforderlich (etwa $0,75 \%$ für 1 IE rekombinanter FIX [BeneFIX $\left.{ }^{\circledR}\right]$ ). Die HWZ beträgt ca. 18 bis $24 \mathrm{~h}$.

Formel zur Dosisberechnung:

KG $(\mathrm{kg}) \times$ gewünschter FIX-Anstieg für pdFIX KG $(\mathrm{kg}) \times$ gewünschter FIX-Anstieg $\times 1,25$ für rFIX

Folgedosen sollten auf Basis von Talspiegeln und der individuellen Recovery und Halbwertszeit angepasst werden. 


\section{Weitere Empfehlung für Operationen/}

\section{Zahnextraktionen}

- Geplante Operationen sollten nur in Zentren mit Erfahrung in der Behandlung von Hämophilie durchgeführt werden (CCHC oder HTC) (Level 3).

- Eine präoperative Inhibitortestung soll erfolgen und ein perioperativer Behandlungsplan vom Hämophiliebehandler erstellt werden (Level 3).

- Für Zahnextraktionen wird eine Faktorsubstitution bis zu einem Spiegel von mindestens $50 \%$ über 2-7 Tage empfohlen. Eine antifibrinolytische Therapie wird für 7 Tage empfohlen. Eine zusätzliche Blutstillung mit Fibrinkleber soll in Erwägung gezogen werden.

- Bei Eingriffen im Schleimhautbereich soll Tranexamsäure zusätzlich verabreicht werden (Level 2).

- Bei leichter Hämophilie A ist die Verabreichung von Desmopressin vor Operationen - unter Beachtung der Kontraindikationen - möglich, wenn ein Ansprechen auf Desmopressin bekannt und ausgetestet ist (Level 3).

- Prä-, peri- und postoperativ wird für Hämophiliepatienten generell keine Thromboseprophylaxe empfohlen.

\section{Therapie bei Trauma und lebensbedrohlichen Blutungen}

Bei schweren Traumata und/oder lebensbedrohlichen Blutungen (z. B. intrazerebrale, schwere gastrointestinale, abdominelle oder retroperitoneale Blutungen, Blutungen im Rachen-oder Halsbereich oder im Auge) ist die Dosierung so zu wählen, dass ein normaler Talspiegel (mindestens 50\%) gehalten wird. Als „loading dose" können die Dosierungsvorgaben verwendet werden, wie sie vor Operationen vorgeschlagen werden. Bei diesen Blutungen gilt: zuerst substituieren, wenn Faktorkonzentrat vorhanden ist, dann evaluieren und transportieren. Der Patient soll möglichst in ein Zentrum mit Hämophilie-Erfahrung transportiert werden, auf jeden Fall muss schon beim Transport darauf Bedacht genommen werden, dass am geplanten Ort der Versorgung Faktorkonzentrat bereits zur Verfügung steht oder schon während des Patiententransports dorthin angeliefert wird. Die Dauer der Substitution wird im Allgemeinen mehrere Tage betragen, bis die Blutung völlig abgeklungen bzw. resorbiert ist. Angaben für anzustrebende Faktorspiegel finden sich in Tab. 3. In den allermeisten Fällen ist die stationäre Aufnahme des Patienten zu empfehlen. Bei Inhibitorpatienten sind „Bypassing Agents“ (APCC $\left(\right.$ FEIBA $^{\circledR}$ )oder rekombinanter Faktor VIIa) in der höchsten empfohlenen Dosierung bzw. im kürzesten empfohlenen Intervall zu wählen.

\section{Empfehlungen Trauma und lebensbedrohliche Blutungen}

- Sofortiges Anheben das Faktorspiegels (siehe Tab. 3) in den Normbereich bei Zeichen einer möglicherweise lebensbedrohlichen Blutung, weitere Dosierung und Dauer nach Evaluation der Blutung (z. B. CT oder MRT) bis zum kompletten Stillstand der Blutung bzw. bis zur Resorption derselben (Level 4).

- Bei Inhibitorpatienten sind „Bypassing Agents“ (APCC oder r-FVIIa) in der höchsten empfohlenen Dosierung bzw. im kürzesten empfohlenen Intervall zu wählen.

- Ein CCHC oder HTC für die Therapieübernahme sollte bevorzugt für die weitere Versorgung gewählt bzw. die Therapie mit einem solchen abgestimmt werden.

\section{Therapie von Patienten mit Inhibitoren} (Hemmkörpern)

Das Risiko, Hemmkörper gegen einen substituierten Faktor zu entwickeln, ist teilweise genetisch bedingt und betrifft etwa 20 bis $30 \%$ aller Patienten mit schwerer Hämophilie A; bei Hämophilie B liegt das Hemmkörperrisiko bei unter $5 \%$. Besonders im Kindesalter treten nicht selten klinisch nicht relevante, transiente Hemmkörper auf, die nach wenigen Wochen spontan wieder verschwinden. Je nach dem Anstieg des Inhibitortiters unterscheidet man sogenannte „low responder“ (<5 BU) und „high responder“ (>5 BU).

Bei diesen Hemmkörpern handelt es sich um Alloantikörper (Ak gegen ein körperfremdes Antigen der eigenen Spezies). Die Bestimmung von Hemmkörpern gegen FVIII sollte nach der Bethesda-Methode in der Nijmegen-Modifikation erfolgen (Level 1) [1, 43, 44].

Bei ca. $50 \%$ der Patienten, die Hemmkörper entwickeln, liegen nur niedrige Hemmkörpertiter vor, die eine Therapie mit höheren Faktoren-Dosierungen oder Verkürzung der Intervalle erlauben. Hohe Hemmkörpertiter neutralisieren hingegen die substituierten Faktorkonzentrate und machen die Behandlung damit ineffektiv. Bei schwerer Hämophilie manifestieren sich Hemmkörper zumeist nach fünf bis 20 Expositionstagen.

\section{Behandlung akuter Blutungen und Blutungsprophylaxe}

Akute Blutungen müssen bei Patienten mit hohen Hemmkörpertitern mittels sogenannter „Bypassing Agents" behandelt werden. Auf dem Markt sind in Österreich zwei derartige Produkte, ein APCC aus Plasma $\left(\right.$ FEIBA $^{\circledR}$ ) und ein rekombinanter Faktor VIIa (Novoseven $^{\circledR}$ ). Beide Präparate können Blutungen stillen bzw. verhindern, wie aus Beobachtungsstudien bekannt ist $[45,46]$. Es gibt jedoch Therapieversager und die Wirk- 
samkeit kann zwischen unterschiedlichen Patienten und bei verschiedenen Blutungskomplikationen variieren. Klinische Vergleichsstudien zeigten bezüglich Wirksamkeit und Sicherheit bisher keine signifikanten Unterschiede zwischen den beiden Substanzen [47].

Die empfohlene Einzeldosis für Novoseven ${ }^{\circledR}$ beträgt $90 \mu \mathrm{g} / \mathrm{kg} \mathrm{KG}$ alle 2-4 h bis zum Stillstand der Blutung. Alternativ kann bei milden bis moderaten Blutungen eine initiale Einzeldosis mit $270 \mu \mathrm{g} / \mathrm{kg} \mathrm{KG}$ verabreicht werden [45]. Die empfohlene Einzeldosis für FEIBA ${ }^{\circledast}$ beträgt $50-100 \mathrm{IE} / \mathrm{kg} \mathrm{KG}$ in 8- bis 12-stündlichen Intervallen nach einer präoperativen Dosis von 75-100 IE/kg KG [46]. Eine Tagesdosis von $200 \mathrm{IE} / \mathrm{kg}$ KG sollte wegen einer Risikoerhöhung für Thrombosen und Myokardinfarkte nicht überschritten werden [46].

Es existiert eine Konsensusempfehlung für den Gebrauch von FEIBA ${ }^{\circledR}$ für Hämophilie-A-Patienten mit Hemmkörpern, die sich elektiven orthopädischen oder nichtorthopädischen Eingriffen unterziehen müssen. Darin sind konkrete Angaben für Dosierungen und Dauer der Therapie nach großen und kleineren Eingriffen angeführt [48]. Für die perioperative Anwendung von Novoseven $^{\circledR}$ wurden 2011 die vorhandenen Daten aus klinischen Studien und Registern zusammengefasst [49].

Hinsichtlich der prophylaktischen Gabe eines der beiden Produkte ist die Datenlage für die Reduktion spontaner Blutungen nicht ausreichend, um eine generelle Empfehlung geben zu können [49-51]. Für beide Produkte konnte eine Verminderung der Blutungsfrequenz gezeigt werden $[50,52]$. Jedoch sind mehr prospektive RCT zur besseren Definition von Behandlungsmodalitäten und optimalen Dosierungen zu fordern.

Patienten mit niedrigem Inhibitortiter (<3-5BU) können mit erhöhten Dosen von Faktorprodukten und/oder kürzeren Therapieintervallen behandelt werden. Im Fall einer Blutung oder Operation sind engmaschige Überwachungen des FVIII-Plasmaspiegels notwendig.

Bei Hämophilie-B-Patienten mit Inhibitor kann es bei Gabe von FIX-Konzentraten zu akuten allergischen Reaktionen und auch zu einem nephrotischen Syndrom bei Immunkomplexnephritis kommen.

\section{Hemmkörperelimination}

Als Immuntoleranztherapie (ITT) ist die tägliche Gabe von hohen Dosen des jeweiligen Faktors definiert, die oft über Monate fortgesetzt werden muss. Sie wird so lange weitergeführt, bis es zu einem Verschwinden des Hemmkörpers kommt, wonach eine schrittweise Reduktion der Faktordosis erfolgt. Die ITT ist bei etwa 60 bis $80 \%$ aller Hämophilie-A Patienten erfolgreich. Bei Hämophilie B ist die Erfolgsrate deutlich geringer - sie liegt um die $30 \%$.

Soweit klinisch vertretbar, wird empfohlen, nach Auftreten des Hemmkörpers zu warten, bis der Hemmkörpertiter auf weniger als 10 Bethesda-Einheiten $/ \mathrm{ml}$ gesunken ist, bevor mit der ITT begonnen wird $[53,54]$.

\begin{tabular}{lll} 
Abkürzungen & \\
\hline Abkürzung & Bedeutung \\
\hline APCC & Aktiviertes Prothrombinkomplexpräparat \\
\hline CCHC & Comprehensive Care Haemophilia Center \\
\hline COX & Cyclooxygenase \\
\hline CT & Computertomographie \\
\hline DDAVP & Desmopressin \\
\hline EBM & Evidenzbasierte Medizin \\
\hline FIX & Faktor IX \\
\hline FVIII & Faktor VIII \\
\hline HTC & Haemophilia Treatment Center \\
\hline HWZ & Halbwertszeit \\
\hline IE & Internationale Einheiten \\
\hline ITT & Immuntoleranztherapie \\
\hline KG & Körpergewicht \\
\hline MRT & Magnetresonanztomographie \\
\hline NSAR & Nichtsteroidale Antirheumatika \\
\hline pd & „Plasma-derived“ \\
\hline PUP & „Previously Untreated Patients“ \\
\hline r & rekombinant \\
\hline RCT & Randomisierte kontrollierte Studie \\
\hline RQW & Rissquetschwunde \\
\hline vWF & Von-Willebrand-Faktor \\
\hline vWS & Von-Willebrand-Syndrom \\
\hline WFH & World Federation of Hemophilia \\
\hline WHO & World Health Organisation \\
\hline & \\
\hline
\end{tabular}

Für die Hämophilie A konnte gezeigt werden, dass eine Hochdosistherapie (200 IE/kg/d) eine kürzere Zeit bis zum Erreichen negativer Titer und einer normalen Recovery sowie niedrigere Blutungsraten mit sich bringt als eine Niedrigdosistherapie (50 IE/kg, 3x/Woche), die beiden Therapieregime jedoch hinsichtlich des Erreichens einer Toleranz nicht unterschiedlich sind [53]. Wenn unter dieser Therapie weiterhin eine Blutungsneigung besteht, kann sie mit Bypassing Agents kombiniert werden [55]. Diese Behandlung wird in der Regel bis einige Monate über das Verschwinden des Hemmkörpers (per definitionem <0,6 Bethesda-Einheiten) hinaus durchgeführt. Eine ITT wird in der Regel mit jenem Produkt begonnen, mit dem der Patient ursprünglich behandelt worden ist. Wenn es allerdings unter diesem Produkt nicht zu einem Abfall der Hemmkörper kommt, sollte die Entscheidung über einen Produktwechsel innerhalb der ersten drei Monate getroffen werden. Eine vielfach angewandte Alternative ist der Wechsel von einem zuvor verwendeten rekombinanten FVIII-Konzentrat auf ein plasmatisches FVIII-Konzentrat mit von-WillebrandFaktor. Ein Vorteil dieses Vorgehens gegenüber dem Beibehalten des ursprünglichen Faktorkonzentrats ist nicht erwiesen.

Der Einsatz von Immunsuppressiva bei Hemmkörpern ist lediglich als zweite Wahl zu sehen. 


\section{Empfehlung für Patienten mit Inhibitor}

- Bypassing Agents (FEIBA ${ }^{\circledR}$; Novoseven ${ }^{\circledR}$ ) sind bei Blutungen und zur Blutungsprophylaxe bei Operationen von Hämophiliepatienten mit Hemmkörpern wirksam. Beide verfügbaren Präparate werden als Therapieoptionen benötigt, da das individuelle Ansprechen unterschiedlich sein kann (Level 2).

- Bei niedrigem Hemmkörpertiter können erhöhte Dosen von Faktorprodukten wirksam sein.

- FEIBA ${ }^{\circledR}$ darf nicht mit Tranexamsäure kombiniert werden. Für Novoseven ${ }^{\circledR}$ wird diese Kombination hingegen empfohlen.

- Eine Immuntoleranztherapie (ITT) ist bei den meisten Patienten mit Hemmkörpern anzustreben (Level 2). Bei Kindern mit niedrigem Hemmkörpertiter und ohne verstärkte Blutungsneigung sollte wegen der Möglichkeit transienter Hemmkörper vor Beginn der ITT einige Wochen zugewartet werden.

- Eine ITT kann mit jenem Produkt begonnen werden, mit dem der Patient ursprünglich auch behandelt worden ist; insbesondere bei NichtAnsprechen kann ein plasmatisches FVIII-Konzentrat mit von-Willebrand-Faktor in Erwägung gezogen werden

\section{Therapie von Konduktorinnen}

Frauen, die genetisch Konduktorinnen der Hämophilie sein könnten, sollten auf ihren FVIII- bzw. FIX-Spiegel untersucht werden, da sie deutlich verminderte Faktorenwerte aufweisen können, die dem Bereich eines Patienten mit leichter Hämophilie entsprechen. Falls dies der Fall ist, erfolgt die Behandlung so wie bei Patienten mit Hämophilie, entsprechend den Faktorenwerten (also z. B. bei leichter Hämophilie A mit Desmopressin oder bei Hämophilie B bzw. im schweren Blutungsfall mit Faktorkonzentraten). Besonderes Augenmerk ist bei Konduktorinnen auf die präoperative Untersuchung und Vorbereitung zu legen (Bestimmung des aktuellen
Faktorwerts, Erstellung eines Behandlungsplans). Im Verlaufe der Schwangerschaft kommt es meist zu einer Normalisierung eines FVIII-, aber weniger eines FIXMangels. Entsprechende Vorkehrungen für die Geburt sollten bei Faktorverminderung getroffen werden, wie z. B. Erstellung eines Behandlungsplans. Die pädiatrischen Hämophilie-Zentren sollten schon während einer Schwangerschaft mit einem möglicherweise hämophilen Kind beigezogen werden.

Auf spezielle Fragestellungen der genetischen Beratung, der vorgeburtlichen Diagnostik und des Geburtsmodus eines hämophilen Kindes wird in dieser Leitlinie nicht eingegangen. Für diese speziellen Situationen sollten immer Zentren mit besonderer Erfahrung (CCHC oder HTC) herangezogen werden.

\section{Danksagung}

Die Autoren danken der Firma Medical Dialogue (GF: Karl Buresch) sowie Dr. med. Norbert Hasenöhrl für ihre Unterstützung bei der Koordination und Organisation dieses Leitlinien-Projekts.

\section{Interessenkonflikt}

Organisation und Durchführung des Leitlinien-Meetings und des nachfolgenden Abstimmungsprozesses sowie die Publikation dieser Leitlinie wurden durch einen Unrestricted Grant der Firma Baxter an die Österreichische Hämophiliegesellschaft (ÖHG) sowie durch Unrestricted Grants der Firmen Bayer, Biotest, CSL Behring, NovoNordisk, Octapharma und Pfizer im Rahmen des Österreichischen Hämophilieregisters an die ÖHG finanziert. Die pharmazeutischen Firmen hatten keinerlei Einfluss auf die Leitlinien und keine Einsicht während des Erstellungsprozesses. Keine(r) der Autorinnen(en) hat für die Erstellung der Leitlinien ein Honorar erhalten.

Open Access Dieser Artikel unterliegt den Bedingungen der Creative Commons Attribution License. Dadurch sind die Nutzung, Verteilung und Reproduktion erlaubt, sofern der/die Originalautor/en und die Quelle angegeben sind. 


\section{Appendix}

Tab. 9 Oxford Centre for Evidence-Based Medicine 2011 Levels Of Evidence. (Quelle: Oxford Centre for Evidence-Based Medicine, hier entnommen aus [1])

\begin{tabular}{|c|c|c|c|c|c|}
\hline Question & Step 1 (Level 1a) & Step 2 (Level 2a) & Step 3 (Levela) & Step 4 (Levela) & Step 5 (Level 5a) \\
\hline $\begin{array}{l}\text { How common is the } \\
\text { problem? }\end{array}$ & $\begin{array}{l}\text { Local and current ran- } \\
\text { dom sample surveys } \\
\text { (or censuses) }\end{array}$ & $\begin{array}{l}\text { Systematic review of sur- } \\
\text { veys that allow matching } \\
\text { to local circumstances }\end{array}$ & $\begin{array}{l}\text { Local nonorandom } \\
\text { sample }^{b}\end{array}$ & Case-series $^{b}$ & $\mathrm{n} / \mathrm{a}$ \\
\hline $\begin{array}{l}\text { Is this diagnostic or } \\
\text { monitoring test accu- } \\
\text { rate? (Diagnosis) }\end{array}$ & $\begin{array}{l}\text { Systematic review of } \\
\text { cross-sectional studies } \\
\text { with consistently } \\
\text { applied reference } \\
\text { standard and blinding }\end{array}$ & $\begin{array}{l}\text { Individual cross-sectional } \\
\text { studies with consistently } \\
\text { applied reference standard } \\
\text { and blinding }\end{array}$ & $\begin{array}{l}\text { Non-consecutive studies, } \\
\text { or studies without } \\
\text { consistently applied } \\
\text { reference standards }^{\text {b }}\end{array}$ & $\begin{array}{l}\text { Case-control studies, or } \\
\text { "poor or nonindependent } \\
\text { reference standard" }\end{array}$ & $\begin{array}{l}\text { Mechanism-based } \\
\text { reasoning }\end{array}$ \\
\hline $\begin{array}{l}\text { What will happen } \\
\text { if we do not add a } \\
\text { therapy? (Prognosis) }\end{array}$ & $\begin{array}{l}\text { Systematic review } \\
\text { of inception cohort } \\
\text { studies }\end{array}$ & inception cohort studies & $\begin{array}{l}\text { Cohort study or control } \\
\text { arm of randomized trial }^{\text {a }}\end{array}$ & $\begin{array}{l}\text { Case-series or case control } \\
\text { studies, or poor quality } \\
\text { prognostic cohort study }\end{array}$ & $\mathrm{n} / \mathrm{a}$ \\
\hline $\begin{array}{l}\text { Does this intervention } \\
\text { help? (Treatment } \\
\text { Benefits) }\end{array}$ & $\begin{array}{l}\text { Systematic review of } \\
\text { randomized trials or } \\
n \text {-of- } 1 \text { trials }\end{array}$ & $\begin{array}{l}\text { Randomized trial or } \\
\text { observational study with } \\
\text { dramatic effect }\end{array}$ & $\begin{array}{l}\text { Non-randomized con- } \\
\text { trolled cohort/follow-up } \\
\text { study }^{b}\end{array}$ & $\begin{array}{l}\text { Case-series, case-control } \\
\text { studies, or historically } \\
\text { controlled studies }^{b}\end{array}$ & $\begin{array}{l}\text { Mechanism-based } \\
\text { reasoning }\end{array}$ \\
\hline $\begin{array}{l}\text { What are the } \\
\text { COMMON harms? } \\
\text { (Treatment Harms) }\end{array}$ & $\begin{array}{l}\text { Systematic review } \\
\text { of randomized trials, } \\
\text { systematic review of } \\
\text { nested case-control } \\
\text { studies, } n \text { of- } 1 \text { trial } \\
\text { with the patient } \\
\text { you are raising the } \\
\text { question about, or } \\
\text { observational study } \\
\text { with dramatic effect }\end{array}$ & $\begin{array}{l}\text { Individual randomized } \\
\text { trial or (exceptionally) } \\
\text { observational study with } \\
\text { dramatic effect }\end{array}$ & $\begin{array}{l}\text { Non-randomized con- } \\
\text { trolled cohort/follow-up } \\
\text { study (postmarketing } \\
\text { surveillance) provided } \\
\text { there are sufficient } \\
\text { numbers to rule out } \\
\text { a common harm. (For } \\
\text { long-term harms the } \\
\text { duration of followup must } \\
\text { be sufficient.) }\end{array}$ & $\begin{array}{l}\text { Case-series, case-control, } \\
\text { or historically controlled } \\
\text { studies }^{b}\end{array}$ & $\begin{array}{l}\text { Mechanism-based } \\
\text { reasoning }\end{array}$ \\
\hline $\begin{array}{l}\text { What are the RARE } \\
\text { harms? (Treatment } \\
\text { Harms) }\end{array}$ & $\begin{array}{l}\text { Systematic review of } \\
\text { randomized trials or } \\
n \text {-of- } 1 \text { trial }\end{array}$ & $\begin{array}{l}\text { Randomized trial or (ex- } \\
\text { ceptionally) observational } \\
\text { study with dramatic effect }\end{array}$ & & & \\
\hline $\begin{array}{l}\text { Is this (early detec- } \\
\text { tion) test worthwhile? } \\
\text { (Screening) }\end{array}$ & $\begin{array}{l}\text { Systematic review of } \\
\text { randomized trials }\end{array}$ & Randomized trial & $\begin{array}{l}\text { Non -randomized con- } \\
\text { trolled cohort/follow-up } \\
\text { study }^{b}\end{array}$ & $\begin{array}{l}\text { Case-series, case-control, } \\
\text { or historically controlled } \\
\text { studies }^{\text {b }}\end{array}$ & $\begin{array}{l}\text { Mechanism-based } \\
\text { reasoning }\end{array}$ \\
\hline \multicolumn{6}{|c|}{$\begin{array}{l}\text { OCEBN1 Levels of Evidence Working Group. „The Oxford } 2011 \text { Levels of Evidence“. Oxford Centre for Evidence-Based Medicine. http://www.cebm.neffindex. } \\
\text { aspx?0 = 5653 } \\
\text { aLevel may he graded down on the basis of study quality, imprecision, indirectness (study PIC0 does not match questions PIC0), because of inconsistency } \\
\text { between studies, or because the absolute effect size is very small; Level may be graded up if there is a large or very large effect size } \\
\text { 'As always, a systematic review is generally better than an individual study }\end{array}$} \\
\hline
\end{tabular}




\section{Literatur}

1. Srivastava A, Brewer AK, Mauser-Bunschoten EP, Key NS, Kitchen S, Llinas A, et al. Guidelines for the management of hemophilia. Haemophilia. 2013;19(1):e1-47.

2. Schulman S. Mild Hemophilia - Revised Edition, November. 2012, No. 41. 2012. http://www.wfh.org/en/page. aspx?pid=1270. Zugegriffen: 9. Sept. 2014.

3. University College London. Factor VIII Variant Database. http://www.factorviii-db.org/. Zugegriffen: 9. Sept. 2014.

4. Colvin BT, Astermark J, Fischer K, Gringeri A, Lassila R, Schramm W, et al. European principles of haemophilia care. Haemophilia. 2008;14(2):361-74.

5. World Federation of Hemophilia (WFH). Compendium of assessment tools. http://www.wfh.org/en/page. aspx?pid=882. Zugegriffen: 10. Jan. 2013.

6. Wissenschaftlicher Beirat der Österreichischen Hämophiliegesellschaft. Konsensus über die Verwendung von Faktorenkonzentraten bei Patienten mit Hämophilie (Version v. 9.11.2012). http://bluter.at/joomla/new_site/images/ stories/Konsensus_9._November2012.pdf. Zugegriffen: 10. Jan. 2013.

7. Kreuz W, Escuriola-Ettingshausen C, Funk M, Schmidt H, Kornhuber B. When should prophylactic treatment in patients with haemophilia A and B start?-The German experience. Haemophilia. 1998;4(4):413-7.

8. Fischer K, van der Bom JG, Molho P, Negrier C, MauserBunschoten EP, Roosendaal G, et al. Prophylactic versus on-demand treatment strategies for severe haemophilia: a comparison of costs and long-term outcome. Haemophilia. 2002;8(6):745-52.

9. Blanchette VS, Shapiro AD, Liesner RJ, Hernandez Navarro F, Warrier I, Schroth PC, et al. Plasma and albumin-free recombinant factor VIII: pharmacokinetics, efficacy and safety in previously treated pediatric patients. J Thromb Haemost. 2008;6(8):1319-26.

10. Collins PW, Blanchette VS, Fischer K, Bjorkman S, Oh M, Fritsch S, et al. Break-through bleeding in relation to predicted factor VIII levels in patients receiving prophylactic treatment for severe hemophilia A. J Thromb Haemost. 2009;7(3):413-20.

11. Collins PW, Bjorkman S, Fischer K, Blanchette V, Oh M, Schroth $\mathrm{P}$, et al. Factor VIII requirement to maintain a target plasma level in the prophylactic treatment of severe hemophilia A: influences of variance in pharmacokinetics and treatment regimens. J Thromb Haemost. 2010;8(2):269-75.

12. Richards $\mathrm{M}$, Williams $\mathrm{M}$, Chalmers E, Liesner R, Collins P, Vidler V, et al. A United Kingdom Haemophilia Centre Doctors' Organization guideline approved by the British Committee for Standards in Haematology: guideline on the use of prophylactic factor VIII concentrate in children and adults with severe haemophilia $\mathrm{A}$. $\mathrm{Br} \mathrm{J}$ Haematol. 2010;149(4):498-507.

13. Keeling D, Tait C, Makris M. Guideline on the selection and use of therapeutic products to treat haemophilia and other hereditary bleeding disorders. A United Kingdom Haemophilia Center Doctors' Organisation (UKHCDO) guideline approved by the British Committee for Standards in Haematology. Haemophilia. 2008;14(4):671-84.

14. Mannucci PM. Hemostatic drugs. N Engl J Med. 1998;339(4):245-53.

15. Frachon X, Pommereuil M, Berthier AM, Lejeune S, Hourdin-Eude S, Quero J, et al. Management options for dental extraction in hemophiliacs: a study of 55 extractions (20002002). Oral Surg Oral Med Oral Pathol Oral Radiol Endod. 2005;99(3):270-5.
16. Franchini M, Rossetti G, Tagliaferri A, Pattacini C, Pozzoli D, Lorenz C, et al. Dental procedures in adult patients with hereditary bleeding disorders: 10 years experience in three Italian Hemophilia Centers. Haemophilia. 2005;11(5):504-9.

17. Hvas AM, Sorensen HT, Norengaard L, Christiansen K, Ingerslev J, Sorensen B. Tranexamic acid combined with recombinant factor VIII increases clot resistance to accelerated fibrinolysis in severe hemophilia A. J Thromb Haemost. 2007;5(12):2408-14.

18. Luu $\mathrm{H}$, Ewenstein B. FEIBA safety profile in multiple modes of clinical and home-therapy application. Haemophilia. 2004;10(Suppl 2):10-6.

19. Giangrande PL, Wilde JT, Madan B, Ludlam CA, Tuddenham EG, Goddard NJ, et al. Consensus protocol for the use of recombinant activated factor VII [eptacog alfa (activated); NovoSeven] in elective orthopaedic surgery in haemophilic patients with inhibitors. Haemophilia. 2009;15(2):501-8.

20. Manco-Johnson MJ, Abshire TC, Shapiro AD, Riske B, Hacker MR, Kilcoyne R, et al. Prophylaxis versus episodic treatment to prevent joint disease in boys with severe hemophilia. N Engl J Med. 2007;357(6):535-44.

21. Gringeri A, Von Mackensen S. Quality of life in haemophilia. Haemophilia. 2008;14(Suppl 3):19-25.

22. Berntorp E, Fischer K, Miners A. Models of prophylaxis. Haemophilia. 2012;18(Suppl 4):136-40.

23. Coppola A, Tagliaferri A, Capua M D, Franchini M. Prophylaxis in children with hemophilia: evidence-based achievements, old and new challenges. Semin Thromb Hemost. 2012;38(1):79-94.

24. Carpenter SL, Soucie JM, Presley RJ, Ragni MV, Wicklund $\mathrm{BM}$, Silvey M, et al. Hepatitis B vaccination is effective by subcutaneous route in children with bleeding disorders: a universal data collection database analysis. Haemophilia. 2015;21(1):e39-43.

25. Kroger AT, Atkinson WL, Pickering LK. 8 - General immunization practices. In: Offit PA, Plotkin SA, Orenstein WA Herausgeber. Vaccines. 6th Aufl. London: W.B. Saunders; 2013. S. 88-112.

26. Aledort LM, Haschmeyer RH, Pettersson H. A longitudinal study of orthopaedic outcomes for severe factor-VIIIdeficient haemophiliacs. The orthopaedic outcome study group. J Intern Med. 1994;236(4):391-9.

27. Abshire TC, Brackmann HH, Scharrer I, Hoots K, Gazengel C, Powell JS, et al. Sucrose formulated recombinant human antihemophilic factor VIII is safe and efficacious for treatment of hemophilia A in home therapy-International Kogenate-FS Study Group. Thromb Haemost. 2000;83(6):811-6.

28. Rodriguez-Merchan EC, Magallon M. The role of a 3-year period of continuous prophylactic concentrate substitution on later development of haemophilic arthropathy. Haemophilia. 1997;3(2):108-10.

29. Khoriaty R, Taher A, Inati A, Lee C. A comparison between prophylaxis and on demand treatment for severe haemophilia. Clin Lab Haematol. 2005;27(5):320-3.

30. Hay CR. Prophylaxis in adults with haemophilia. Haemophilia. 2007;13(Suppl 2):10-5.

31. Tagliaferri A, Franchini M, Coppola A, Rivolta GF, Santoro C, Rossetti G, et al. Effects of secondary prophylaxis started in adolescent and adult haemophiliacs. Haemophilia. 2008;14(5):945-51.

32. Walsh CE, Valentino LA, Factor VIII. prophylaxis for adult patients with severe haemophilia A: results of a US survey of attitudes and practices. Haemophilia. 2009;15(5):1014-21.

33. Quon DV, Konkle BA. How we treat: haematuria in adults with haemophilia. Haemophilia. 2010;16(4):683-5. 
34. Ghosh K, Jijina F, Mohanty D. Haematuria and urolithiasis in patients with haemophilia. Eur J Haematol. 2003;70(6):410-2.

35. Singleton T, Kruse-Jarres R, Leissinger C. Emergency department care for patients with hemophilia and von Willebrand disease. J Emerg Med. 2010;39(2):158-65.

36. Bush MT, Roy N. Hemophilia emergencies. J Emerg Nurs. 1995;21(6):531-8 (quiz 8-40).

37. Blanchette VS, Key NS, Ljung LR, Manco-Johnson MJ, van den Berg HM, Srivastava A. Definitions in hemophilia: communication from the SSC of the ISTH. J Thromb Haemost. 2014;12(11):1935-9.

38. Negrier C, Shapiro A, Berntorp E, Pabinger I, Tarantino M, Retzios A, et al. Surgical evaluation of a recombinant factor VIII prepared using a plasma/albumin-free method: efficacy and safety of Advate in previously treated patients. Thromb Haemost. 2008;100(2):217-23.

39. Hermans C, Altisent C, Batorova A, Chambost H, De Moerloose P, Karafoulidou A, et al. Replacement therapy for invasive procedures in patients with haemophilia: literature review, European survey and recommendations. Haemophilia. 2009;15(3):639-58.

40. Piot B, Sigaud-Fiks M, Huet P, Fressinaud E, Trossaert M, Mercier J. Management of dental extractions in patients with bleeding disorders. Oral Surg Oral Med Oral Pathol Oral Radiol Endod. 2002;93(3):247-50.

41. Zanon E, Martinelli F, Bacci C, Zerbinati P, Girolami A. Proposal of a standard approach to dental extraction in haemophilia patients. A case-control study with good results. Haemophilia. 2000;6(5):533-6.

42. Björkman S, Berntorp E. Pharmacokinetics of coagulation factors: clinical relevance for patients with haemophilia. Clin Pharmacokinet. 2001;40(11):815-32.

43. Meijer P, Verbruggen B. The between-laboratory variation of factor VIII inhibitor testing: the experience of the external quality assessment program of the ECAT foundation. Semin Thromb Hemost. 2009;35(8):786-93.

44. Verbruggen B, van Heerde WL, Laros-van Gorkom BA. Improvements in factor VIII inhibitor detection: from Bethesda to Nijmegen. Semin Thromb Hemost. 2009;35(8):752-9.

45. Young G, Cooper DL, Gut RZ. Dosing and effectiveness of recombinant activated factor VII (rFVIIA) in congenital haemophilia with inhibitors by bleed type and location: the experience of the Haemophilia and Thrombosis Research Society (HTRS) Registry (2004-2008). Haemophilia. 2012;18(6):990-6.
46. Hilgartner M, Aledort L, Andes A, Gill J. Efficacy and safety of vapor-heated anti-inhibitor coagulant complex in hemophilia patients. FEIBA Study Group. Transfusion. 1990;30(7):626-30.

47. Astermark J, Donfield SM, DiMichele DM, Gringeri A, Gilbert SA, Waters J, et al. A randomized comparison of bypassing agents in hemophilia complicated by an inhibitor: the FEIBA NovoSeven Comparative (FENOC) Study. Blood. 2007;109(2):546-51.

48. Rangarajan S, Austin S, Goddard NJ, Negrier C, RodriguezMerchan EC, Stephensen D, et al. Consensus recommendations for the use of $\operatorname{FEIBA}(\mathrm{R})$ ) in haemophilia A patients with inhibitors undergoing elective orthopaedic and nonorthopaedic surgery. Haemophilia. 2013;19(2):294-303.

49. Valentino LA, Cooper DL, Goldstein B. Surgical experience with rFVIIa (NovoSeven ${ }^{\circledR}$ ) in congenital haemophilia A and B patients with inhibitors to factors VIII or IX. Haemophilia. 2011;17(4):579-89.

50. Ettingshausen CE, Kreuz W. Early long-term FEIBA prophylaxis in haemophilia A patients with inhibitor after failing immune tolerance induction: a prospective clinical case series. Haemophilia. 2010;16(1):90-100.

51. Franchini M, Manzato F, Salvagno GL, Montagnana M, Zaffanello M, Lippi G. Prophylaxis in congenital hemophilia with inhibitors: the role of recombinant activated factor VII. Semin Thromb Hemost. 2009;35(8):814-9.

52. Konkle BA, Ebbesen LS, Erhardtsen E, Bianco RP, Lissitchkov T, Rusen L, et al. Randomized, prospective clinical trial of recombinant factor VIIa for secondary prophylaxis in hemophilia patients with inhibitors. J Thromb Haemost. 2007;5(9):1904-13.

53. Hay CR, DiMichele DM. The principal results of the International Immune Tolerance Study: a randomized dose comparison. Blood. 2012;119(6):1335-44.

54. Dimichele D. The North American Immune Tolerance Registry: contributions to the thirty-year experience with immune tolerance therapy. Haemophilia. 2009;15(1):320-8.

55. Oldenburg J. [Immune tolerance therapy for inhibitors in haemophilia A]. Hämostaseologie. 2008;28(Suppl 1):S23-5.

56. Endler G, Slavka G, Perkmann T, Haushofer A. [The importance of preanalytics for the coagulation laboratory]. Hämostaseologie. 2010;30(2):63-70.

57. Knöbl P, Spannagel M. Erworbene Hemmkörper-Hämophilie. Stuttgart: Ligatur-Verlag; 2009. 